\title{
Increase in vertical dimension and maxillo-mandibular growth in a longitudinal growth sample
}

\author{
S. Akimoto, M. Kubota, S. Sato \\ Division of Orthodontics, Department of Craniofacial Growth and Development Dentistry, Kanagawa Dental College, \\ Kanagawa, Japan
}

Received December 12, 2009; Accepted December 8, 2009

Introduction: The relationship between the increase in vertical dimension (VD) and growth of the maxilla and mandible is poorly established. In order to examine the relationship between VD change and growth of the maxillo-facial complex, we measured the vertical dental and skeletal heights, and maxillo-mandibular growth.

Materials and methods: The study material consisted of serial lateral cephalograms of 25 subjects in whom the cephalographic series was complete, taken every year from the age of 6-14 years along with radiographs and impressions. We examined growth in different stages, Stage 1 through 4 .

Results: The correlation analysis between the increase in dental and skeletal VD and the forward growth of maxilla and mandible showed that the growth of maxilla and mandible was significantly correlated with the increase in skeletal VD (S-PP) as well as dental VD. The mandibular growth in the Class III group was highly correlated with the increase in dental VD.

Conclusions: From these findings, it was concluded that maxillo-mandibular growth is strongly related to VD.

Keywords: Vertical dimension (VD), maxilla, mandible, growth, longitudinal sample

\section{Introduction}

Humans exhibit a wide variety of craniofacial morphology, and they consequently develop different types of malocclusion. Orthodontic management of skeletal malocclusion, Classes II and III can often be frustrating. The necessity of early intervention has long been recognized, although there are still no effective treatment modalities in the early stage of craniofacial growth. This may be because the developmental processes of these malocclusions are not yet understood. Considerable changes in skeletal and dental vertical dimension (VD) take place during the transition phase from deciduous dentition to permanent dentition, and malocclusion often develops during this period.

Correspondence: Sadao Sato, Division of Orthodontics, Department of Craniofacial Growth and Development Dentistry, Kanagawa Dental College, 82 Inaoka-cho, Yokosuka 238-8580, Japan.

E-mail: satos@kdcnet.ac.jp
The changes in the vertical height of dentition and in the occlusal plane during growth greatly affect the growth of the craniomandibular skeleton and the development of skeletal malocclusion $[8,9]$. When the vertical height of the dentition is not sufficient and the occlusal plane maintains a steep inclination during growth and development, the lower jaw is forced rearwards and Class II occlusion develops. Excessive increase in vertical height of the dentition and a flat occlusal plane are factors that encourage protrusive adaptation of the mandible, and Class III occlusion develops $[10,11]$.

In the present study, the relationships between the increase of skeletal and dental VD and growth of the maxilla and mandible were examined using longitudinal growth samples.

\section{Materials and methods}

In this study, cephalometric radiographs collected during a 14-year period from 1960 and 1974 at the Department of Craniofacial Growth and Development Dentistry, Kanagawa Dental College were used. Twenty-five subjects were selected based on the following criteria: 1) a series of dental casts which clearly showed the occlusion during the entire period covering the transition to permanent dentition, 2) no orthodontic or prosthetic treatment, and 3) no destruction of the dentition and occlusion by oral disease or trauma. Each subject had at least 9 pairs of upper and lower dental casts (at about 1-year intervals) and at least 9 cephalometric radiographs (also at about 1-year intervals). Each cephalometric radiograph was traced in the customary way and lengths and angles were measured to analyze the relationships between the VD increase and craniofacial morphology. We checked the growth in the stages of 6-year-molar eruption (Stage 1), beginning of exchange buccal segment (Stage 2), end of exchange buccal segment (Stage 3), and completion of permanent teeth occlusion (Stage 4).

\section{Classifying growth patterns}

For judging the growth patterns of the craniofacial complex, the anteroposterior dysplasia indicator (APDI) [5] was used. 
The APDI measures the facial-plane angle and the angle which is the sum of the $\mathrm{AB}$ plane angle and FH-PP. It is the index used for evaluating the anteroposterior character of the craniofacial complex. Skeletal Classes I, II, and III correspond to APDI readings of $78-84^{\circ},<77^{\circ}$, and $>85^{\circ}$, respectively. The three sample groups consisted of 9 subjects with Class I, 7 subjects with Class II, and 9 subjects with Class III.

\section{Cephalometric measurements related to maxillo-mandibular and VD (Fig. 1)}

\section{Measurement of VD}

The change in the VD was measured. VD is defined as the length of the perpendicular line from the center of the mesiodistal width of the upper first molar to the palatal plane (PP) (U6-PP) and the center of the mesio-distal width of the lower molar to the mandibular plane (MP) (L6-MP). In addition, to quantify the change in skeletal VD, the distance from sella turcica (S) to the PP was measured (S-PP). Total dental VD increase was expressed as U6 plus L6 (U6+ L6). Total VD increase is the sum of U6-PP, L6-MP, and S-PP.

\section{Forward growth of maxilla and mandible}

The change in the anteroposterior growth of maxilla and mandible was determined by drawing a perpendicular line from the lowest point of the fossa pterygopalatina (Ptm) to the PP and then measuring the distance from there to the most anterior point of the maxilla (A) and to the most anterior point of the mandible (B).

\section{Relationship between VD change and maxillo-mandibular growth}

The changes in VD and the increase in maxillo-mandibular forward growth were measured and calculated for each

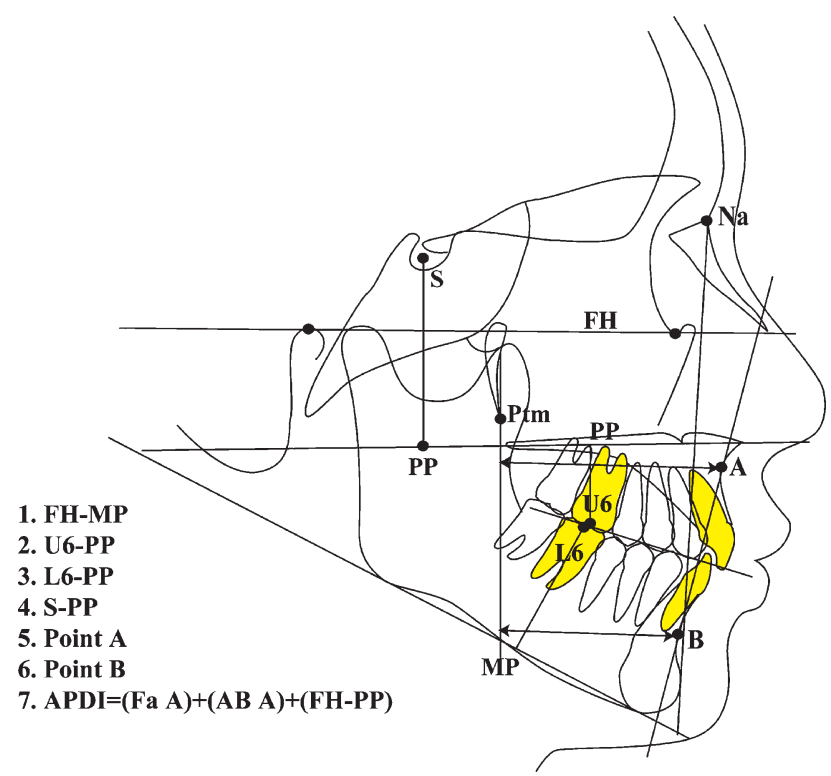

Fig. 1: Cephalometric analysis of the craniofacial morphology and vertical dimension growth interval, and any correlation between these variables was recorded.

\section{Statistical analysis}

The correlation between increase in VD and forward growth of the maxilla and mandible was assessed by Pearson's correlation analysis. The significance of correlation was assessed at the $5 \%$ and $1 \%$ levels. Statistical analysis was performed using SPSS (version 5).

\section{Results}

Correlation between the growth of maxilla and mandible and the increase of VD in Class I group

The maxillary growth (Point A) in the Class I group was significantly correlated with S-PP and total VD (Table 1). The mandibular growth (Point B) was also significantly correlated with S-PP and total VD. Growth of the maxilla and mandible in this group was not correlated with dental VD (U6-PP, L6-MP, and U6 + L6).

\section{Correlation between anteroposterior growth of maxilla} and mandible and increase of VD in Class II Group

The maxillary growth (Point A) in the Class II group was significantly correlated only with S-PP (Table 2), while the mandibular growth (Point B) was significantly correlated with S-PP and total VD. Growth of the maxilla and mandible was not correlated with dental VD (U6-PP, L6-MP, and U6 + L6).

Tab. 1: Correlation between maxillo-mandibular growth and increment of vertical dimension in Class I group

\begin{tabular}{lll}
\hline & $\begin{array}{l}\text { Correlation } \\
\text { Coefficient }\end{array}$ & $\begin{array}{l}\text { Significance } \\
(\boldsymbol{P} \text {-value })\end{array}$ \\
\hline Point A vs. & & \\
\hline U6-PP & 0.339 & 0.084 \\
\hline L6-MP & 0.117 & 0.561 \\
\hline U6 + L6 & 0.319 & 0.105 \\
\hline S-PP & 0.429 & $0.026^{*}$ \\
\hline Total VD & 0.438 & $0.022^{*}$ \\
\hline Point B & 0.476 & $0.012^{*}$ \\
\hline
\end{tabular}

\section{Point B vs.}

\begin{tabular}{lll}
\hline U6-PP & 0.228 & 0.253 \\
\hline L6-MP & 0.204 & 0.309 \\
\hline U6 + L6 & 0.290 & 0.142 \\
\hline S-PP & 0.534 & $0.004^{* *}$ \\
\hline Total VD & 0.480 & $0.011^{*}$ \\
\hline *Significant correlation at $P<0.5 ;{ }^{* *}$ significant correlation at $P<0.1$ \\
\hline
\end{tabular}


Tab. 2: Correlation between anteroposterior jaw growth and increment of vertical dimension in Class II

\begin{tabular}{ll}
$\begin{array}{l}\text { Correlation } \\
\text { coefficient }\end{array}$ & $\begin{array}{l}\text { Significance } \\
(P \text {-value })\end{array}$ \\
\hline
\end{tabular}

\section{Point A vs.}

\begin{tabular}{lll}
\hline U6-PP & 0.006 & 0.979 \\
\hline L6-MP & 0.195 & 0.397 \\
\hline U6 + L6 & 0.139 & 0.547 \\
\hline S-PP & 0.564 & $0.008^{* *}$ \\
\hline Total VD & 0.348 & 0.122 \\
\hline Point B & 0.341 & 0.131 \\
\hline
\end{tabular}

Point B vs.

\begin{tabular}{lll}
\hline U6-PP & 0.430 & 0.052 \\
\hline L6-MP & 0.302 & 0.184 \\
\hline U6 + L6 & 0.412 & 0.063 \\
\hline S-PP & 0.470 & $0.032^{*}$ \\
\hline Total VD & 0.503 & $0.020^{*}$ \\
\hline * Significant correlation at $P<0.5 ;{ }^{* *}$ significant correlation at $P<0.1$ \\
\hline
\end{tabular}

Tab. 3: Correlation between anteroposterior jaw growth and increment of vertical dimension in Class III

$\begin{array}{ll}\begin{array}{l}\text { Correlation } \\ \text { coefficient }\end{array} & \begin{array}{l}\text { Significance } \\ (P \text {-value })\end{array}\end{array}$

\section{Point A vs.}

\begin{tabular}{lll}
\hline U6-PP & 0.556 & $0.003^{* *}$ \\
\hline L6-MP & 0.462 & $0.015^{*}$ \\
\hline U6 + L6 & 0.582 & $0.001^{* *}$ \\
\hline S-PP & 0.198 & 0.321 \\
\hline Total VD & 0.601 & $0.001^{* *}$ \\
\hline Point B & 0.563 & $0.002^{* *}$ \\
\hline
\end{tabular}

Point B vs.

\begin{tabular}{lll}
\hline U6-PP & 0.611 & $0.001^{* *}$ \\
\hline L6-MP & 0.375 & 0.054 \\
\hline U6 + L6 & 0.568 & $0.002^{* *}$ \\
\hline S-PP & 0.273 & 0.168 \\
\hline Total VD & 0.627 & $0.000^{* *}$ \\
\hline *Significant correlation at $P<0.5 ;{ }^{* *}$ significant correlation at $P<0.1$ \\
\hline
\end{tabular}

Correlation between anteroposterior growth of maxilla and mandible and increase of VD in Class III group

The maxillary growth (Point A) was significantly correlated with U6-PP, U6-MP, U6 + L6, and total VD, but not with S-PP
(Table 3). The mandibular growth (Point B) in Class III was significantly correlated with U6-PP, U6 + L6, and total VD, but not with S-PP.

\section{Correlation between forward growth of the mandible} and increase of VD in Class III group

Although not likely with Classes I and II, the mandibular growth (Point B) in Class III was more significantly related to dental VD than to skeletal VD. The correlations between the mandibular forward growth (Point $\mathrm{B}$ ) and VD increase (U6 + L6, U6-PP, and Total VD) are shown in Figs. 2 through 4 .

\section{Class III}

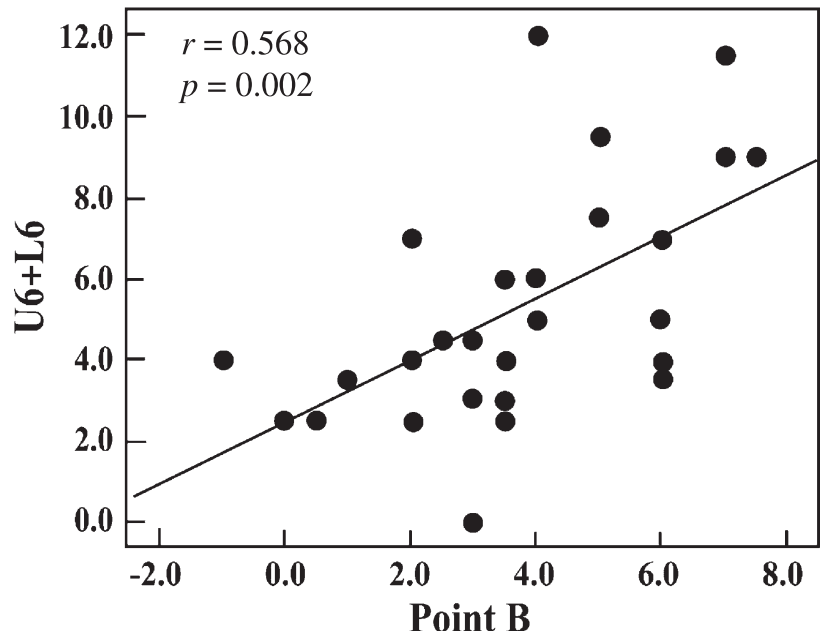

Fig. 2: Correlation between dental VD and mandibular forward growth in the Class III group. There was strong correlation between the VD increase of upper and lower 1st molars and the mandibular forward growth (Point B) with 0.568 of correlation coefficient, significant at $P=0.002$ leve

\section{Class III}

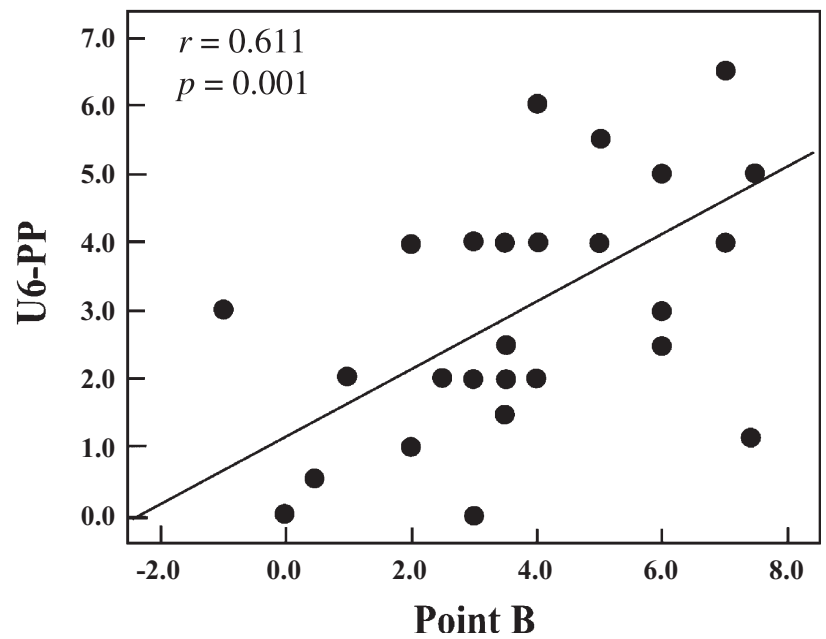

Fig. 3: Correlation between the dental VD and mandibular forward growth in the Class III group. There was strong correlation between the increase of U6-PP and the mandibular forward growth (Point B) with 0.611 of correlation coefficient, significant at $P=0.001$ level 


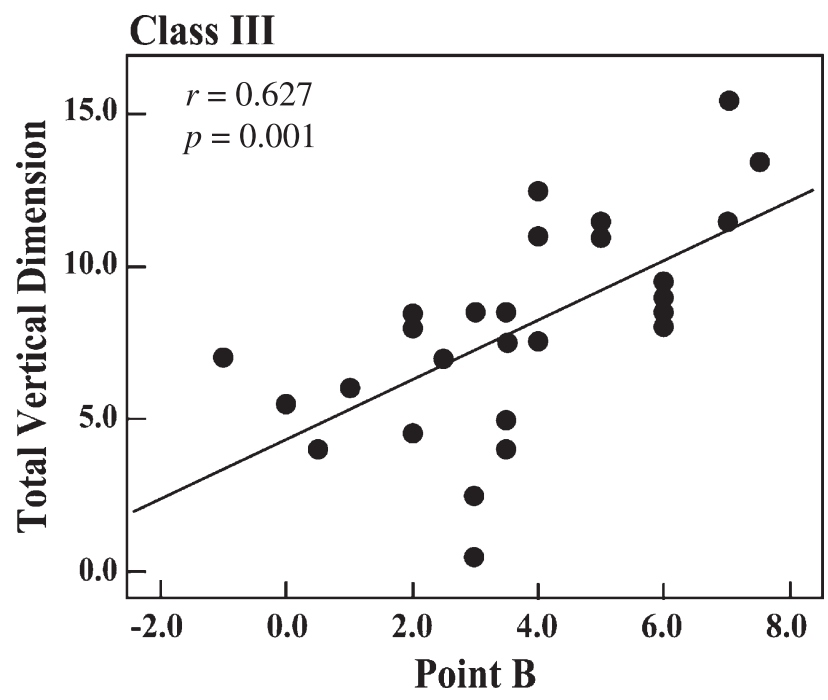

Fig. 4: Correlation between the total VD and mandibular forward growth in the Class III group. There was strong correlation between the increase of total VD and the mandibular forward growth (Point B) with 0.627 of correlation coefficient, significant at $P<0.001$ level

\section{Discussion}

How the dental and skeletal VD influences craniofacial growth is a subject of fundamental interest to researchers in the fields of Pedodontics and Orthodontics. In particular, the influence of $\mathrm{VD}$, including occlusion, on the growth of the mandible is the essential element by which we may understand how to control facial growth and, more importantly, to prevent malocclusion. In the present study, we examined the relationship between the dental and skeletal VD and maxillo-mandibular growth using longitudinal growth data from a sample drawn from among native Japanese.

The results show that all groups, Classes I, II, and III, had significant correlation with total VD increase, except point A against VD in Class II group. In Class I and Class II groups, Point A and Point B were significantly correlated with skeletal VD (S-PP), however not with dental VD. The Class III group, had correlation with dental VD such as U6-PP and U6 + L6 as well as total VD, however not with skeletal VD (S-PP). These results show that in Class III development, dental VD has more influence than skeletal VD, while other groups show more influence from skeletal VD.

The correlation between the Point B growth and dental and VD in the Class III group was significant (Figs. 2-4), indicating that the increase in dental VD influences the mandibular forward growth. Sato [9] reported that the occlusal plane in skeletal Class III malocclusion becomes flatter during growth and development. Mandibular forward adaptation is important in the developmental process of skeletal Class III malocclusion. During the growing process, posterior discrepancy influences the increasing dental VD by pushing out molars. The close relationship between the increase in dental VD and mandibular forward growth in the present results also shows some effect from posterior discrepancy.

Hwang et al. [2,3] reported that the inclination of the posterior occlusal plane was closely related to the anteroposterior relationship of the mandible. In addition, Kato et al. [6] and Fushima et al. [1] reported the vertical dimension and inclination of the occlusal plane of Class II malocclusion cases; the vertical dimension of the molar was significantly lower and the occlusal plane was steeper. In addition, the series of investigations conducted by our group [4, 7-13] showed that VD and occlusal plane change during growth and adaptation of the mandible, which is an important element of craniofacial skeletal growth, and the cause of malocclusion. In addition, it indicates that the retrognathic mandible, such as in Class II malocclusion, is the result of secondary adaptation of the mandible [14].

From these previous reports together with the present results, it is clear that mandibular forward growth is related to dental and skeletal VD changes. It also indicates that early treatment of malocclusion should be directed to regulate dental VD, especially in Class III development.

\section{Conclusions}

Based on the present study of the VD and maxillo-mandibular growth in a longitudinal growth sample, the following conclusions can be made.

1) In Class I and Class II groups, there is a significant correlation between skeletal VD and maxillo-mandibular growth.

2) In the Class III group, there was a strong correlation of dental VD increase and mandibular forward growth.

These results indicate that changes in VD greatly influence the growth of the mandible. In addition, change in VD during growth and development contributes to manifestation of skeletal malocclusion.

\section{Take-home message}

The increase in vertical dimension (VD) and growth of the maxilla and mandible are closely related. In particular, the mandibular growth of the Class III group was highly correlated with the increase in dental VD.

\section{Acknowledgments}

This work was performed at the Research Institute of Occlusion Medicine and Research Center of Brain and Oral Science, Kanagawa Dental College, and supported by a Grant-in-Aid for Open Research from the Ministry of Education, Culture, Sports, Science and Technology, Japan.

\section{Conflict of interest}

The authors declare that there is no conflict of interest.

\section{References}

[1] Fushima K, Kitamura Y, Mita H, Sato S, Suzuki Y, Kim YH. Significance of the cant of occlusal plane in Class II division 1 malocclusion. Eur J Orthodont 1996;18:27-40.

[2] Hwang DH, Akimoto S, Sato S. Occlusal plane and mandibular posture in the hyperdivergent type of malocclusion in mixed dentition subjects. Bull Kanagawa Dent Coll 2002;30:87-92. 
[3] Hwang DH, Akimoto S, Sato S. Relationship between the occlusal plane inclination and mandibular posture in the hyperdivergent type of skeletal frame. Bull Kanagawa Dent Coll 2003;31:39-49.

[4] Kim J II, Akimoto S, Shinji H, Sato S. Importance of vertical dimension and cant of occlusal plane in craniofacial development. Int J Stomat Occ Med 2009;2:114-21.

[5] Kim YH, Vietas JJ. Anteroposterior dysplasia indicator: an adjunct to cephalometric differential diagnosis. Am J Orthodont 1978;73:619-35.

[6] Kato S, Chung WN, Kim JI, Sato S. Morphological characterization of high and low angle types of Class II malocclusion. Bull Kanagawa Dent Coll 2002;30:93-8.

[7] Sato S, Sasaguri K, Kamoi S, Goto M, Suzuki Y. Importance of posterior tooth-to-denture base discrepancy in the development of skeletal open-bite malocclusion (Japanese with English abstract). J Jpn Orthod Soc 1990;49:322-30.

[8] Sato S, Suzuki Y. Relationship between the development of skeletal mesio-occlusion and posterior tooth-to-denture base discrepancy Its significance in the orthodontic reconstruction of skeletal class III malocclusion. J Jpn Orthod Soc 1988;47:796-810.
[9] Sato S. Case report: Developmental characterization of skeletal Class III malocclusion. Angle Orthodontist 1994;64:105-12.

[10] Sato S, Motoyanagi K, Suzuki T, Imasaka S, Suzuki Y. Longitudinal study of the denture frame changes and its relationship with the development of skeletal malocclusions (Japanese with English abstract). J Jpn Orthod Soc 1988;47:186-96.

[11] Sato S, Suzuki N, Suzuki Y. Longitudinal study of the cant of the occlusal plane and the denture frame in cases with congenitally missing third molars - further evidence for the posterior discrepancy. J Jpn Orthod Soc 1988;47:517-25.

[12] Sato S, Kim J II, Kim K-M, Tokiwa O, Yoshimi H, Onodera K, Sasaguri K. Significance of early orthodontic treatment of malocclusion with dysfunction in the craniomandibular system. Bull Kanagawa Dent Coll 2004;32:37-48.

[13] Tanaka EM, Sato S. Longitudinal alteration of the occlusal plane and development of different dentoskeletal frames during growth. Am J Orthod Dentofacial Orthop 2008;134:602, e1-11.

[14] Verrela J. Early developmental traits in Class II malocclusion. Acta Odont Scand 1998;56:375-7. 